\title{
Salud indígena en Brasil: pluralismo médico y autoatención
}

\author{
ESTHER JEAN LANGDON
}

Indigenous Health in Brasil:

Medical Pluralism and

Self-Attention

ESTHER JEAN LANGDON

Instituto Nacional de Ciência e Tecnologia

Brasil Plural, Universidade Federal

de Santa Catarina, Florianópolis, Brasil

estherjeanbr@gmail.com

Traducción: Mauricio Pardo Rojas

Desacatos 58,

septiembre-diciembre 2018, pp. 8-15 $\mathrm{n}$ varios países del mundo, la antropología médica internacional se constituyó con una influencia profunda de la producción estadounidense, basada en estudios orientados a la biomedicina. A su vez, la tradición médico-antropológica en Latinoamérica tiene un núcleo de producción teórica que se combina con estudios etnográficos sólidos, relacionados con temas y dinámicas locales, en particular en situaciones culturalmente diferenciadas. De acuerdo con Seppilli y Otegui, lo que distingue a las investigaciones "latinoamericanas" en antropología médica es su enfoque metodológico, su análisis e interpretación, ya que pretenden aludir "más directamente a las desigualdades y a las macro-lógicas de poder de los sistemas sociales y del proceso de globalización planetaria. El problema, en efecto, no es de escala de los fenómenos analizados, pese a la inevitable diversidad en los procedimientos de investigación, sino de enfoque metodológico en su examen e interpretación" (2005: 8).

Los enfoques latinoamericanos proporcionaron una evaluación crítica de los límites etnocéntricos del modelo biomédico, sin descuidar la importancia que éste tiene en el mundo contemporáneo ni descartar las transacciones y el uso de servicios de tipo biomédico por parte de las personas. Esta mirada permitió reflexionar sobre la estructuración de las políticas públicas y las condiciones de salud de las poblaciones más vulnerables, y combinó el punto de vista de los sujetos con los análisis de las condiciones de vida estructurales.

En ese sentido, se destaca el carácter fundamental de los trabajos de Eduardo Menéndez, quien desde la década de 1980 impulsó una producción guiada por una perspectiva crítica en el área de la antropología médica; generó conceptos clave para el campo, como "modelo médico hegemónico" y "autoatención”, y defendió el método etnográfico clásico y la reflexión sobre el concepto de medicina tradicional. 
Menéndez empezó sus contactos con Brasil a finales de la década de 1990, sobre todo por su vínculo con el movimiento de medicina social y salud colectiva en Latinoamérica. Esos intercambios con la antropología y la salud en Brasil comenzaron con su participación en conferencias colectivas sobre el tema. En 1998, sus artículos aparecieron en publicaciones nacionales (Menéndez, 1998; 2003; 2016; Menéndez y Di Pardo, 2005; Trabalho, Educação e Saúde, 2012). Dado que la mayoría estaba en español, en 2009 se publicó una colección en portugués de sus artículos principales para hacerlos accesibles (Menéndez, 2009).

Su perspectiva alcanzó ciertas corrientes de pensamiento en el campo de la salud colectiva en Brasil, el cual se ha centrado por tradición en los ámbitos políticos y legislativos del sistema de salud, con pocas reflexiones sobre las condiciones concretas de vida de la población y sus representaciones y prácticas de autoatención. Sin embargo, el curso institucionalista que siguió la reforma sanitaria brasileña dio predominio al debate sobre los modelos biomédicos de atención, las estrategias de gobernabilidad y la gestión de la relación entre los tres niveles de gobierno - federal, estatal y municipal-.

Por otro lado, los antropólogos brasileños han utilizado cada vez más los conceptos de Menéndez para entender la dinámica de la pluralidad médica en el país y para analizar la reforma de salud y el Sistema Único de Salud (sus) de Brasil, que tienen como principios el acceso universal a la salud y se basan en la participación democrática y el respeto a la diversidad cultural. Estas investigaciones se orientan de acuerdo con los modelos teórico-metodológicos desarrollados por Menéndez y otros investigadores interesados en una perspectiva antropológica capaz de entender la dimensión emic de la enfermedad y la salud, sin sacrificar por ello las dinámicas establecidas por el proceso de colonización y las relaciones asimétricas presentes en contextos interculturales.
La sección temática de este número de Desacatos tiene como objetivo presentar una muestra de las investigaciones que se producen en Brasil y que reflejan la influencia de Menéndez. Son el resultado de la colaboración de larga data entre antropólogos y profesionales de la salud dedicados a la investigación de la salud indígena y el activismo. Participantes activos en la construcción de la política de salud indígena y preocupados por la importancia de la investigación etnográfica para la evaluación de los servicios de salud en contextos interculturales (Langdon y Garnelo, 2017), su investigación refleja lo que puede caracterizarse como el enfoque crítico brasileño. Están comprometidos en la investigación estratégica que tiene la intención de beneficiar a las comunidades investigadas e informar a los responsables de la construcción de las políticas públicas. Defienden el método etnográfico que establece el diálogo y reconoce el pluralismo del conocimiento (Montardo y Rufino, 2017). Se esfuerzan por crear no sólo un diálogo con las comunidades indígenas estudiadas, sino también entre la antropología, la salud y las políticas públicas (Garnelo y Langdon, 2005).

En 2009, los autores se incorporaron al Instituto Nacional de Ciencia y Tecnología Brasil Plural (IBP), en el marco del proyecto "Prácticas de autoatención, redes, itinerarios y políticas públicas". El grupo ha podido intensificar sus colaboraciones con otros países de Latinoamérica, en particular con México y Colombia, pero también con Venezuela, Argentina y Chile (Langdon y Cardoso, 2015). Estos investigadores participan en discusiones metodológicas y teóricas relacionadas con el desarrollo de conceptos adecuados para la diversidad de Latinoamérica y la comprensión del poder y la autonomía locales, que se expresan tanto en la praxis de los itinerarios terapéuticos como en las relaciones entre los servicios biomédicos hegemónicos y las poblaciones a las que sirven. 


\section{Subsistema indígena brasileño}

El Subsistema de Salud Indígena fue creado en 1999 para extender los servicios de salud a unos 200 grupos indígenas de Brasil, ubicados en todo el país. Hasta su creación, la política de salud para los pueblos indígenas estaba mal definida y faltaban servicios adecuados para estos grupos, que se distinguían por sus lenguas y culturas, así como por su grado de contacto con la sociedad en general. El Subsistema estableció 34 distritos de salud en el país para la prestación de atención primaria en los territorios indígenas - Terras Indígenas - ${ }^{1}$ En cada distrito, los centros administrativos llamados Polo Base son responsables de la provisión de servicios de salud. Equipos interdisciplinarios -Equipe Médica de Saúde Indígena (EMSI)—, que consisten idealmente en médicos, enfermeras, técnicos y agentes indígenas de salud (AIS), son contratados para servir a las comunidades. Como subsistema del sus, los pacientes indígenas que requieren servicios más especializados son remitidos a la red mayor de éste.

La Política nacional de atención a la salud para los pueblos indígenas garantiza el acceso universal a los servicios primarios y la provisión de atención médica que respeta las particularidades culturales. En el sistema brasileño, la noción de interculturalidad se expresa como "atención diferenciada" y se refiere al subsistema diferenciado basado en los distritos de salud, así como a la atención diferenciada por profesionales de la salud capacitados que respetan la eficacia de la medicina indígena y "el derecho de estos pueblos a su cultura” (Fundação Nacional de Saúde, 2002: 13). El documento que establece los estados de la política nacional dice que "el principio que impregna todas las directivas de la Política nacional de atención a la salud de los pueblos indígenas es el respeto a las concepciones, valores y prácticas relativas a los procesos de salud y enfermedad de cada sociedad indígena y sus diversos especialistas" (2002: 18).
En otra parte, el documento caracteriza los sistemas de salud indígenas tradicionales:

Como basados en un enfoque holístico de la salud, cuyo principio es la armonía de las personas, las familias y las comunidades con el universo que los rodea. Las prácticas de curación responden a la lógica interna de cada comunidad indígena y son el producto de la relación particular con el mundo espiritual y los seres en el entorno en el que viven. Estas prácticas y concepciones son, en general, recursos de salud de eficacia empírica y simbólica, de acuerdo con la definición más reciente de salud de la Organización Mundial de la Salud (2002: 17).

Se describen tres vías para la atención diferenciada: capacitación de recursos humanos para el trabajo en contextos interculturales, articulación con sistemas de salud indígenas tradicionales y capacitación de AIS para estimular la apropiación por los pueblos indígenas de los conocimientos y técnicas de la medicina occidental, "no como una sustitución, sino como una adición a la colección de sus propias terapias y otras prácticas culturales, ya sean tradicionales o no" (2002: 16).

\section{Los artículos}

Los artículos de este dosier examinan las prácticas médicas tradicionales, la interacción entre pacientes y profesionales de la salud, y el papel de los AIS y su relación con los profesionales de la salud y las comunidades a las que sirven. Se han adoptado varios conceptos de Eduardo Menéndez (1992; 2003), como

Terra Indígena es la designación del territorio indígena reconocido de manera legal y demarcado como "ancestral" por el gobierno brasileño. 
modelos de atención médica, prácticas de autoatención y modelo médico hegemónico. Los artículos demuestran que los grupos caracterizados como rurales y "tradicionales" tienen acceso a una serie de modelos de atención de la salud y que articulan, incorporan y se apropian de elementos de cada uno para formar una hibridación dinámica de las prácticas. El enfoque relacional de Menéndez subraya el reconocimiento de pluralismos médicos y recursos terapéuticos más allá de la biomedicina y la agencia de grupos sociales en la búsqueda de la prevención y resolución de problemas de salud. El concepto de prácticas de autoatención contrarresta la tendencia a caracterizar la medicina tradicional o indígena y la etnomedicina como homogéneas y estáticas.

La intermedicalidad es otro concepto desarrollado por la red, presente en los análisis publicados aquí. Similar a la perspectiva de Menéndez, el término apunta a resaltar los aspectos políticos, económicos e ideológicos involucrados en las interacciones entre profesionales médicos y grupos indígenas, examina las asimetrías de relación y cómo los miembros del grupo subordinado incorporan y se apropian de sustancias y prácticas biomédicas. Este concepto, originalmente sugerido por Shane Greene (1998), fue presentado en Brasil por MajLis Follér (2004) para analizar la asimetría en las relaciones y la hibridez de las prácticas en la zona de contacto entre el equipo de Médicos Sin Fronteras y los indígenas shipibo-conibo. Follér indica que la intermedicalidad señala que la expansión de los servicios biomédicos entre los pueblos indígenas es una zona de contacto en la que la realidad social está constituida por negociaciones entre sujetos políticamente activos y reconoce que todos los actores involucrados exhiben agencia social.

La manera de utilizar los conceptos de intermedicalidad y prácticas de autoatención en estos artículos demuestra, contra las expectativas, que la biomedicina no reemplaza las formas indígenas de conocimiento y prácticas, a pesar de su expansión continua. La investigación etnográfica basada en estos conceptos dirige la atención a las dinámicas corporales, sociales y cosmológicas involucradas en la reproducción biosocial en su sentido más amplio, y demuestra que la salud debe entenderse en una perspectiva más amplia, que reconoce la pluralidad y dinámica de los sistemas de conocimiento y de procesos relacionados con el cuerpo, con formas de sociabilidad, cosmología y ontología.

Las prácticas de autoatención entre los munduruku, descritas por Raquel Paiva Dias-Scopel y Daniel Scopel, exhiben la profundidad y la importancia del conocimiento local en el manejo de la salud materna, que funciona mediante una red de actores que incluye a las mujeres mayores de la familia, especialistas en partería y practicantes sagrados, a los que se recurre cuando se detectan complicaciones con causas o motivos ocultos. Los conceptos de los munduruku sobre el cuerpo y el proceso de salud/enfermedad/atención no pueden separarse de la más amplia naturaleza cosmopolítica de su visión del mundo y su sistema de curación. Aunque las mujeres munduruku buscan ayuda del programa gubernamental de salud materna cuando creen que es necesario, siguen siendo relativamente autónomas y no abandonan sus prácticas de autoatención, a pesar de los esfuerzos de los programas de salud materna para controlar la experiencia del embarazo.

Los estudios sobre el pueblo kaingang del sur de Brasil, una región alejada de la Amazonia, muestran que las prácticas de autoatención de este grupo son extremadamente dinámicas, ya que aprovechan todos los modelos de atención disponibles.

Los indígenas de las Tierras Indígenas Kaingang, en el sur de Brasil, una de las regiones agroindustriales más ricas del país, se caracterizan por ser un grupo en contacto e interacción constantes con la sociedad nacional. Ari Ghiggi Junior presenta la trayectoria de Luciana como sanadora. Su articulación entre religiones y modelos de atención de la salud, así como su circulación en las ciudades cercanas 


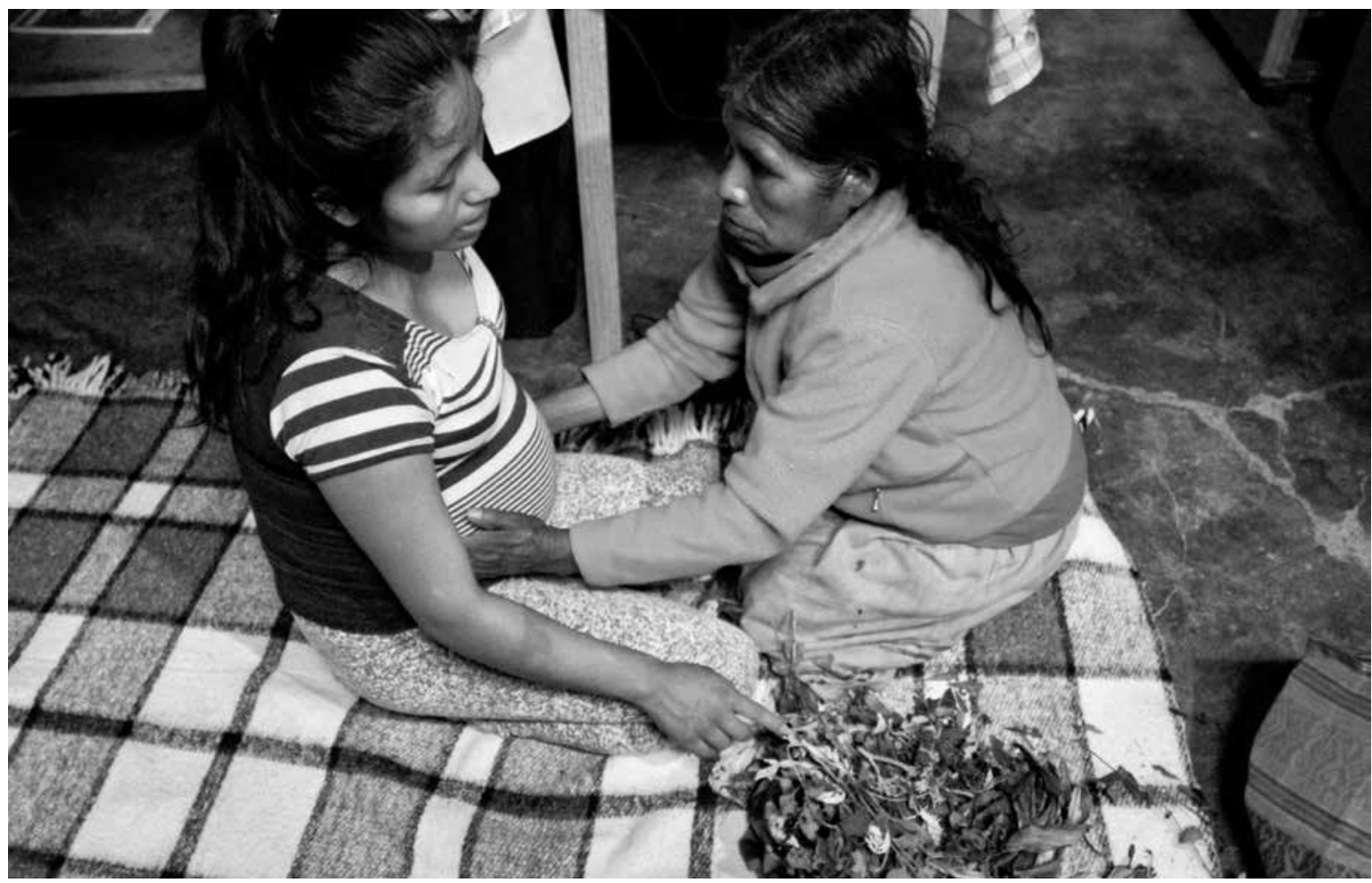

Consuelo Morales Pagaza - Nieves, partera, emplea plantas medicinales para atender a las mujeres antes, durante y después del parto. Zoltecomapa,

Acatepec, Guerrero, 2017.

para atender clientes no indígenas, desafían las características estereotipadas de la medicina tradicional.

Sandra Carolina Portela García examina las prácticas de autoatención entre los pacientes kaingang diagnosticados con diabetes y presión arterial alta. Como los munduruku, sus percepciones sobre los síntomas y la experiencia de la enfermedad se basan en una perspectiva más amplia del cuerpo, el medio ambiente y la religión. Las descripciones etnográficas del programa gubernamental implementado para atender a estos pacientes y de la interacción entre los miembros del equipo de salud y los pacientes kaingang demuestran que la comunicación es asimétrica, lineal y unidireccional. A pesar de que la política de salud indígena exige la capacitación de profesionales médicos con el fin de respetar el conocimiento nativo y las prácticas nativas en estos entornos interculturales, las enfermeras que ejecutan el programa de diabetes entre los kaingang ignoran los comentarios y las percepciones de sus pacientes indígenas. Las interacciones son un claro ejemplo de hegemonía comunicativa por parte de los profesionales de la salud, un concepto asociado a los procesos del modelo médico hegemónico (Menéndez y Di Pardo, 1996, citado en Briggs, 2005).

Los hallazgos de Portela García se replican en el estudio etnográfico de Dassuem Nogueira y Luiza Garnelo, sobre prácticas de autocuidado diseñadas para tratar el paludismo endémico en una población rural de la Amazonia. La comunidad tiene sus propias representaciones en cuanto a la importancia de esta enfermedad prevalente y mantiene sus explicaciones respecto a su prevención, síntomas, causas y consecuencias para la vida social. La sintomatología 
de la malaria se reordena y resignifica, y da como resultado la percepción de variedades de malaria que no corresponden al diagnóstico biomédico. Como en el programa de diabetes entre los kaingang, las relaciones de poder impregnan la interacción entre los líderes comunitarios, los pacientes y sus familias con los profesionales encargados de llevar a cabo acciones de control sobre la enfermedad. Al mismo tiempo, la definición social de las variedades de malaria también se relaciona con las luchas intracomunitarias por el poder y la influencia sobre los programas gubernamentales.

El trabajo sobre AIS en la región del Alto Rio Negro, de Ana Lúcia Pontes, Sergio Rego y Luiza Garnelo, tiene un enfoque más explícito sobre la mediación cultural o interculturalidad. El Subsistema de Salud Indígena fue creado para garantizar "acceso universal, articulación con conocimientos y prácticas indígenas, y participación ciudadana en todas las fases de planificación, ejecución y evaluación de acciones de salud" (Fundação Nacional de Saúde, 2002: 8). Respecto a la participación, se creó la figura del AIs para desarrollar formas de mediación entre tradiciones médicas, aumentar la participación indígena en los servicios de salud y contribuir a la calidad de los servicios interculturales. El papel de los AIS se entiende como el vínculo entre el conocimiento tradicional y el biomédico, entre la comunidad y el equipo de salud. El AIS se percibe como un participante intercultural en la práctica de la atención diferenciada. El texto contribuye a los estudios de otros miembros de la red para evaluar el éxito del AIS como un articulador entre las prácticas médicas y sus relaciones con los equipos de salud (Langdon, Diehl y Dias-Scopel, 2014). Los autores demuestran que el AIS no es reconocido como un profesional de la salud debido a la naturaleza jerárquica y burocrática de los equipos médicos interdisciplinarios y que los miembros del equipo dan poco valor a las prácticas médicas tradicionales, lo que se opone a los principios de atención diferenciada. Sin embargo, el papel del AIs como actor que contribuye de manera potencial a la expansión de la medicalización se ve atenuado por la autonomía de la comunidad para combinar los recursos terapéuticos disponibles. En el curso de sus esfuerzos para resolver problemas de salud, los miembros de la comunidad buscan al AIS para acceder a servicios biomédicos específicos, en particular para la provisión de remedios alopáticos. En este sentido, la función del AIS es menos de mediador entre sistemas culturales y más de facilitador de la articulación de las prácticas de autoatención de los pacientes y sus familias con las de la biomedicina, entre ellas, la automedicación. En este sentido, sus acciones representan una resistencia al proceso de medicalización entre los grupos indígenas.

\section{Estudios estratégicos y etnografía}

En un importante artículo sobre la relación entre antropología y salud colectiva, Maria Cecília de Souza Minayo (1998) separa lo que considera etnografías básicas de los estudios estratégicos. Para ella, los estudios etnográficos básicos examinan la salud como parte de las relaciones políticas, económicas y domésticas inherentes a la organización social de un grupo con poca vinculación directa a cuestiones políticas más amplias, en consecuencia, tienden a permanecer dentro del dominio académico. Por otro lado, los estudios estratégicos proponen comprender las condiciones de producción de las actividades de los servicios de salud y evaluar las relaciones y los programas institucionales. En su opinión, para la implementación de políticas públicas, la antropología es más relevante en la segunda dimensión. Los estudios presentados aquí indican que la distinción entre las etnografías antropológicas básicas y los estudios estratégicos no es tan clara cuando está orientada por una perspectiva que reconoce la naturaleza política de las interacciones micro. Las investigaciones sobre las redes de curación de 
los kaingang, las prácticas de embarazo de los munduruku o los agentes indígenas de salud de los baniwa demuestran con claridad que las prácticas médicas tradicionales no representan costumbres del pasado condenadas a desaparecer con la expansión de la biomedicina. Por el contrario, son muy significativas para las políticas públicas, que tienen como objetivo proporcionar servicios en un contexto intercultural.

Las producciones académicas brasileñas que utilizan los conceptos de autoatención e intermedicalidad en el contexto de la salud indígena en Brasil señalan su potencial para las investigaciones en los campos de la antropología de la salud y la salud colectiva. También resaltan las dinámicas creativas que se encuentran en las prácticas de autoatención y desafian la dicotomía generalizada entre los modelos de atención indígenas y los oficiales. Todas las investigaciones convergen en que, a pesar de su hegemonía y del apoyo que recibe por parte del Estado por medio del Subsistema de Salud Indígena, la biomedicina no suplanta otras formas de prácticas terapéuticas. Por el contrario, la expansión continua de sus servicios y medicamentos coexiste con el florecimiento de modelos populares y alternativos de atención médica.
Los estudios reunidos en este dosier tienen relevancia más allá de Brasil y su política de salud indígena. Sus hallazgos pueden relacionarse con el contexto más amplio de la pluralidad médica y la diversidad cultural característica de Latinoamérica. La atención diferenciada, como se define en la Política nacional de atención a la salud de los pueblos indígenas (Fundação Nacional de Saúde, 2002), exige reconocimiento y respeto a las prácticas y el conocimiento indígena, así como la articulación o incorporación de sus prácticas. Por lo general, en el continente, este principio se conoce como "interculturalidad" y encierra la noción de "una relación entre culturas dinámicas, en la cual existe necesariamente reciprocidad, voluntad y horizontalidad" (Hasen, 2012). La perspectiva de Menéndez (2016) sobre las prácticas de autoatención e intermedicalidad, expresada en su modelo teórico conceptual, contribuye a la construcción de un enfoque crítico latinoamericano que refuerza la naturaleza estratégica y oportuna de las descripciones etnográficas, en la medida en que éstas enfocan la interculturalidad desde la perspectiva local y la naturaleza política de las prácticas e interacciones en torno a la salud, ya sean intracomunitarias o entre pacientes indígenas y profesionales en los programas de salud de Brasil. D

\section{Bibliografía}

Briggs, Charles, 2005, "Perspectivas críticas de salud y hegemonía comunicativa: aperturas progresistas, enlaces letales", en Revista de Antropología Social, vol. 14, pp. 101-124.

Follér, Maj-Lis, 2004, "Intermedicalidade: a zona de contato criada por povos indígenas e profissionais de saúde", en Esther Jean Langdon y Luiza Garnelo (orgs.), Saúde dos povos indígenas: reflexões sobre antropologia participativa, Associação Brasileira de Antropologia/ ContraCapa, Río de Janeiro, pp. 103-116.

Fundação Nacional de Saúde, 2002, Política nacional de atenção à saúde dos povos indígenas, Ministério da Saúde, Brasilia.

Garnelo, Luiza y Esther Jean Langdon, 2005, "A antropologia e a reformulação das práticas sanitárias na atenção básica à saúde”, en Maria Cecília de Souza Minayo y Carlos E. A. Coimbra Jr. (orgs.), Críticas e atuantes: ciências sociais e humanas em saúde na América Latina, Fundação Oswaldo Cruz, Río de Janeiro, pp. 133-156.

Greene, Shane, 1998, “The Shaman's Needle: Development, Shamanic Agency, and Intermedicality in Aguaruna Lands, Peru”, en American Ethnologist, vol. 25, núm. 4, pp. 634-658.

Hasen Narváez, Felipe Nayip, 2012, "Interculturalidad en salud: competencias en práticas de salud con población indígena”, en Ciencia y Enfermería, vol. 18, núm. 3, pp. 17-24. 
Langdon, Esther Jean y Marina Cardoso (orgs.), 2015, Saúde indígena: políticas comparadas na América Latina, Editora de la Universidade Federal de Santa Catarina (Coleção Brasil Plural), Florianópolis.

Langdon, Esther Jean y Luiza Garnelo (orgs.), 2004, Saúde dos povos indígenas: reflexões sobre antropologia participativa, Associação Brasileira de Antropologia/ContraCapa, Río de Janeiro.

, 2017, "Articulación entre servicios de salud y 'medicina indígena': reflexiones antropológicas sobre política y realidad en Brasil”, en Salud Colectiva, vol. 13, núm. 3, pp. 457-470. Disponible en línea: <http://dx.doi.org/10.18294/sc.2017.1117>.

Langdon, Esther Jean, Eliana E. Diehl y Raquel Paiva Dias-Scopel, 2014, "O papel e a formação dos agentes indígenas de saúde na atenção diferenciada à saúde aos povos indígenas brasileiros”, en Carla Teixeira y Luiza Garnelo (orgs.), Saúde indígena en perspectiva: explorando suas matrizes históricas e ideológicas, Fundação Oswaldo Cruz, Río de Janeiro, pp. 213-240.

Menéndez, Eduardo, 1992, "Modelo hegemónico, modelo alternativo subordinado, modelo de autoatención. Caracteres estructurales”, en Roberto Campos (comp.), La antropología médica en México, Universidad Autónoma Metropolitana, México, pp. 97-113. , 1994, “La enfermedad y la curación. ¿Qué es medicina tradicional?”, en Alteridades, vol. 4, núm. 7, pp. 71-83.

, 1998, “Antropología médica e epidemiologia. Processo de convergência ou processo de medicalização?”, en Paulo C. Alves y Miriam C. Rabelo (orgs.), Antropologia da saúde. Traçando identidade e explorando fronteiras, Relume Dumará, Río de Janeiro, pp. 71-94.

,2003, "Modelos de atención de los padecimientos: de exclusiones teóricas y articulaciones prácticas", en Ciência \& Saúde Coletiva, vol. 8, núm. 1, pp. 185-208.

, 2009, Sujeitos, saberes e estruturas: uma introdução ao enfoque relacional no estudo da saúde coletiva, Hucitec, São Paulo.

2016, "Salud intercultural: propuestas, acciones y fracasos", en Ciencia \& Saude Coletiva, vol. 21, núm. 1, pp. 109-118.

Menéndez, Eduardo y Renée B. Di Pardo, 2005, "Alcoholismo, otras adicciones y varias imposibilidades", en Maria Cecília de Souza Minayo y Carlos E. A. Coimbra Jr. (orgs.), Críticas e atuantes: ciências sociais e humanas em saúde na América Latina, Río de Janeiro, Fundação Oswaldo Cruz, pp. 567-586.

Minayo, Maria Cecília de Souza, 1998, “Construção da identidade da antropologia na área de saúde: o caso brasileiro”, en Paulo C. Alves y Miriam C. Rabelo (orgs.), Antropologia da saúde. Traçando identidade e explorando fronteiras, Relume Dumará, Río de Janeiro, pp. 29 -47.

Montardo, Deise Lucy y Márcia Regina Rufino, 2017, Saberes e ciência plural: diálogos e interculturalidade em antropologia, Editora de la Universidade Federal de Santa Catarina (Coleção Brasil Plural), Florianópolis.

Seppilli, Tullio y Rosario Otegui, 2005, "Antropología médica crítica: presentación”, en Revista de Antropología Social, vol. 14, pp. 7-13.

Trabalho, Educação e Saúde, 2012, “Entrevista: Eduardo Luis Menéndez Spina”, vol. 10, núm. 2, pp. 335-345. 